\title{
Stripping off of the hydration shells in the double layer formation: water networks matter
}

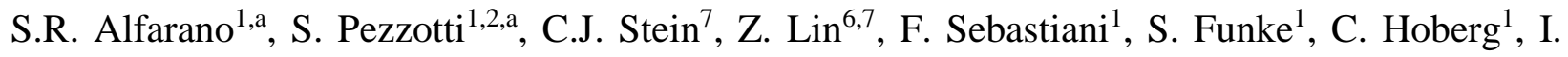 \\ Kolling ${ }^{1}$, C.Y. Ma ${ }^{1}$, K. Mauelshagen ${ }^{1}$, T. Ockelmann ${ }^{1}$, G. Schwaab ${ }^{1}$, L. Fu ${ }^{3}$, J.-B. Brubach ${ }^{4}$, P.

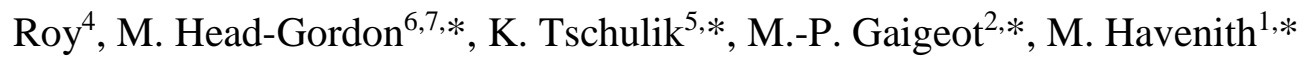 \\ ${ }^{1}$ Lehrstuhl für Physikalische Chemie II, Ruhr-Universität Bochum, 44780 Bochum, Germany \\ ${ }^{2}$ LAMBE UMR8587, Université d'Evry val d'Essonne, CNRS, CEA, Université Paris-Saclay, 91025, Evry, France \\ ${ }^{3}$ Univ Lyon, Univ Claude Bernard Lyon 1, CNRS, Institut Lumière Matière, F-69622 Villeurbanne, France \\ ${ }^{4}$ Synchrotron SOLEIL, AILES beamline, L’Orme des Merisiers, Saint Aubin, BP 48, 91192 Gif sur Yvette \\ Cedex, France \\ ${ }^{5}$ Ruhr University Bochum, Faculty of Chemistry and Biochemistry, Chair of Analytical Chemistry II, Bochum, \\ Germany \\ ${ }^{6}$ Joint Center for Artificial Photosynthesis, Lawrence Berkeley National Laboratory, Berkeley, California \\ 94720, United States \\ ${ }^{7}$ Department of Chemistry, University of California, Berkeley, Berkeley, California 94720, United States
}

${ }^{\text {a }}$ These authors contributed equally: S.R. Alfarano, S. Pezzotti

*email: $\quad \underline{\text { mhg@cchem.berkeley.edu; }} \quad \underline{\text { kristina.tschulik@,rub.de; } \quad \text { mgaigeot@univ-evry.fr; }}$ martina.havenith@,rub.de 


\section{ABSTRACT}

The double layer at the solid/electrolyte interface is a key concept in electrochemistry. Here, we present an experimental study combined with simulations, which provides a molecular picture of the double-layer formation in

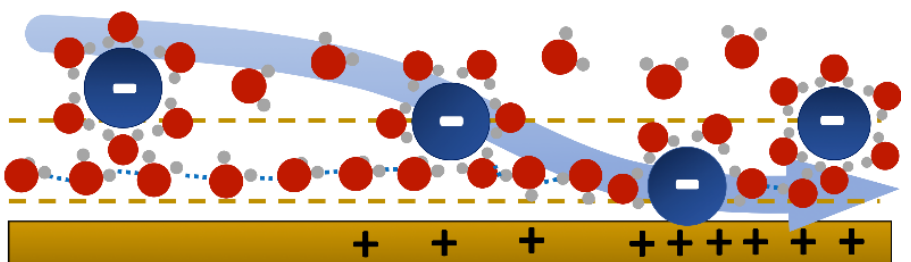
operando processes. By $\mathrm{THz}$ spectroscopy we are able to follow the stripping off of the cation/anion hydration shells for a $\mathrm{NaCl}$ electrolyte at the $\mathrm{Au}$ surface when decreasing/increasing the bias potential. While $\mathrm{Na}^{+}$is attracted toward the electrode already at the smallest applied negative potentials, stripping-off of the $\mathrm{Cl}^{-}$hydration shell is observed only at higher potential values. These phenomena are directly measured by in operando THz spectroscopy with ultra-bright synchrotron light as a source and rationalized by accompanying molecular-dynamics simulations and electronic-structure calculations. 


\section{INTRODUCTION}

One of the most challenging global frontiers is the economic transition towards renewable energy technologies and recycling of waste into valuable chemicals. Most routes to tackle these efforts and develop "green" processes involve electrochemistry. These range from battery, supercapacitor and fuel cell technologies (1), to corrosion and solar cells (2), to electrochemical water splitting,(3) all the way to using electric power for selective reduction of $\mathrm{CO}_{2}$ to form synthetic renewable fuels and valuable chemicals (4). Each of these applications involves electron transfer across the electrode/solution interface and is therefore governed by interfacial chemistry. Thus, all major efforts rely on increasing the speed and selectivity of interfacial reactions. This holds for electrocatalysis, as much as for batteries.

The fundamental principles governing interfacial chemistry have been established decades ago. Marcus theory and the Butler-Volmer formalism describe the general principles of electrochemical reaction kinetics, based either on looking at the solvation of reactants, intermediates and products or on the activated complex formed upon electron transfer, respectively. In parallel, the MottSchottky equation for the depletion layer allows to predict on macroscopic bases the capacitive behaviour at the semiconductor/electrolyte interface (5). In reality, however, very little is known at the molecular level on the structure and solvation state of the reacting species and the way they are activated directly at the electrode, due to lack of a microscopic description of the double layer arising at the solid/electrolyte interface. Several experiments (6-8) showed the validity of the Gouy-Chapman-Stern-Grahame model (GCS) for the description of solid/electrolyte interfaces at a macroscopic level. According to the GCS model (9), the charged electrode is in contact with the Inner Helmholtz Plane (IHP), composed of dehydrated immobile ions specifically bound to the surface. The Outer Helmholtz Plane (OHP), consisting of hydrated ions, follows the IHP, which is 
the diffusive layer with mobile ions. Per contra, on the microscopic scale, the interfacial solvent structure and ion solvation in the IHP/OHP are not explicitly treated, and deviations from the GCS model have been reported $(10,11)$. Whatever the electro-catalytic process, the reactants must approach the surface to react, i.e. they have to penetrate the double layer. A microscopic understanding of the double layer and the role of the solvent (water in most electrochemical applications as of today) in mediating ion-surface interactions is therefore of fundamental importance for future applications.

This requires new experimental techniques that are able to selectively probe the double layer and simultaneously provide molecular-level information, under in operando electrochemical conditions. In the past two decades, a number of theoretical (12-17) and experimental (18-27) studies have been performed to explore the microscopic structure of the electrichemical double layer. However, only recent techniques allow the study of key desolvation/resolvation processes. It is still an experimental challenge to probe interfaces under operando conditions, facing difficulties in regulating all the variables involved in electrochemical reactions $(28,29)$, such as controlling surface structure and mass transport. Charge transfer at an unperturbed double layer has been characterized by an electrochemical probe (30). Using IR spectroscopy, Yamakata et al. (24) investigated modifications in the ion hydration shells on a CO-covered Pt electrode. Local and intramolecular mode probes have been used in Sum Frequency Generation experiments at aqueous interfaces in operando conditions (31) or in SERS/Stark effect spectroscopies (32).

Previous simulation studies further revealed that the hydration of metal surfaces induces the formation of a strongly bound adlayer where water molecules form a 2D-hydrogen bond network parallel to the surface. This adlayer interacts unfavourably with the adjacent water layer, which hence shows hydrophobic features similar to the water-air interface (33). When a negative 
potential is applied, interfacial water reorients with $\mathrm{H}$ atoms toward the gold surface, slightly disrupting the hydrogen bonding network in the interfacial layer (21). These structural changes are expected to have an impact on the electric double layer formation, since the adsorption of ions at the metal surface was shown to require that an adlayer water molecule vacates its adsorption site (13).

In this work we focus on changes in the water network, such as the formation and stripping off of the hydration shell during the formation of the electric double layer (EDL). Any changes in the hydrogen bond network as well as ion hydration can be sensitively probed by $\mathrm{THz}$ spectroscopy, i.e. in the intermolecular fingerprint region between $10-700 \mathrm{~cm}^{-1}$ (34). The low frequency absorption spectrum of bulk water is well known (34-37). Modes around $80 \mathrm{~cm}^{-1}$ are characterized by concerted motions involving the second solvation shell. Cross-correlating these modes with the local dipole demonstrates that their integrated contribution to the total IR activity is indeed very weak, which is consistent with the lack of any resonance around $80 \mathrm{~cm}^{-1}$ in the $\mathrm{THz}$ absorption spectrum, while the peak is present in Optical Kerr as well as Raman spectra. The hydrogen-bond stretching modes contribute to a peak centred at $200 \mathrm{~cm}^{-1}$, which is dominated by first-shell dynamics. The frequency region above $500 \mathrm{~cm}^{-1}$ is dominated by water librational modes, i.e. hindered rotations.

We present the first reported fingerprint of the double-layer formation of an electrolyte solution at a gold surface under applied bias potential using the ultra-bright synchrotron Soleil as a low frequency radiation source. As sample, we used a $10 \mathrm{mM} \mathrm{NaCl}$ solution and applied a positive/negative potential at the gold $(\mathrm{Au})$-liquid interface. The low frequency spectra of aqueous $\mathrm{NaCl}$ have been extensively analysed in detail elsewhere $(38,39)$. Any modification of the hydration layer of the two ions will be clearly identified by the change in their well-known $\mathrm{THz}$ 
fingerprints.

\section{RESULTS}

Following the pioneering work of Nemes et al. (40), we adopted the electrochemical cell developed at AILES (Soleil Facility) (41), consisting of a Au grid as working electrode and a thin platinum foil as counter electrode. Since no Faradaic reactions were driven (see Fig. S1 in SI), we did not employ a reference electrode to prevent concentration alterations of the sample. The potential values reported here are referenced to a "reference potential" $\left(\mathrm{V}_{\text {ref }}\right)$ at which a zero current is measured, equal to $+0.03 \mathrm{~V}$ and $+0.07 \mathrm{~V}$ for the positive and negative potential series, respectively (see Section $\mathrm{S} 8$ of the SI for conversion to a $\mathrm{Ag} / \mathrm{AgCl}$ reference electrode). The exact position of the Au potential of zero charge (PZC) for our specific experimental setup cannot be precisely determined due to the polycrystalline nature of our electrode and the specific electrolyte composition and concentration. However, a recent study by Wang et al. (42) sets the PZC of a polycrystalline $\mathrm{Au}$ electrode in the range $0.17 \mathrm{~V}-0.22 \mathrm{~V}$ vs SHE by SECCM measurements, compatible with the voltage range probed in this present work. We recorded absorption $\mathrm{THz}$ spectra in the $50-350 \mathrm{~cm}^{-1}$ region, varying the potential in $20 \mathrm{mV}$ steps from $0 \mathrm{~V}$ to $+0.2 \mathrm{~V}$ for the positive voltages, and at the following values for the negative voltages: $-2 \mathrm{mV},-4 \mathrm{mV},-8 \mathrm{mV},-10$ $\mathrm{mV},-20 \mathrm{mV},-50 \mathrm{mV},-150 \mathrm{mV}$. In order to confirm that background variations are negligible, the acquisition of each spectrum taken at the potential $V_{i}$ was followed by acquisition of a spectrum at the reference potential $V_{\text {ref. }}$ Fig. 1 shows the difference spectra obtained by referencing each spectrum at potential $V_{i}$ to the spectrum at the lowest or highest applied bias potential for the negative (A) or positive (B) potential series, respectively. This choice allows to better visualise the trends upon voltage application. A crucial advantage is that, in the difference spectra, any spectral contribution 
that does not change with the potential provides by construction a zero intensity. Therefore, the intensity of the difference spectra only arises from the region of the EDL (with a Debye length of $3 \mathrm{~nm}$ ), where the potential is non-zero and decay with increasing distance from the surface, while the bulk does not provide spectral contribution, since it is not affected by the potential. The signal to noise ratio depends on the probed interfacial volume and it is estimated to be $2 \cdot 10^{-4}$ (cfr section $\mathrm{S} 3$ of the SI). All details about the experimental set-up are given in the SI (section S1-S8).

For negative bias potentials, the spectral intensity sharply decreases for potentials of a few millivolts (from $-2 \mathrm{mV}$ to $-8 \mathrm{mV}$ ) and saturates around zero already at $-50 \mathrm{mV}$. This implies that the spectral intensity does not change anymore from $-50 \mathrm{mV}$ up to the most negative potential. A different trend emerges for positive voltages: the spectral intensity initially increases with increasing potential from $0 \mathrm{mV}$ to $+80 \mathrm{mV}$ and starts to decrease only at higher potential values. In order to decipher the reason behind the different behaviour with negative and positive potentials and to unveil the spectral components responsible for the observed trends, we performed a Principal Component Analysis (PCA).

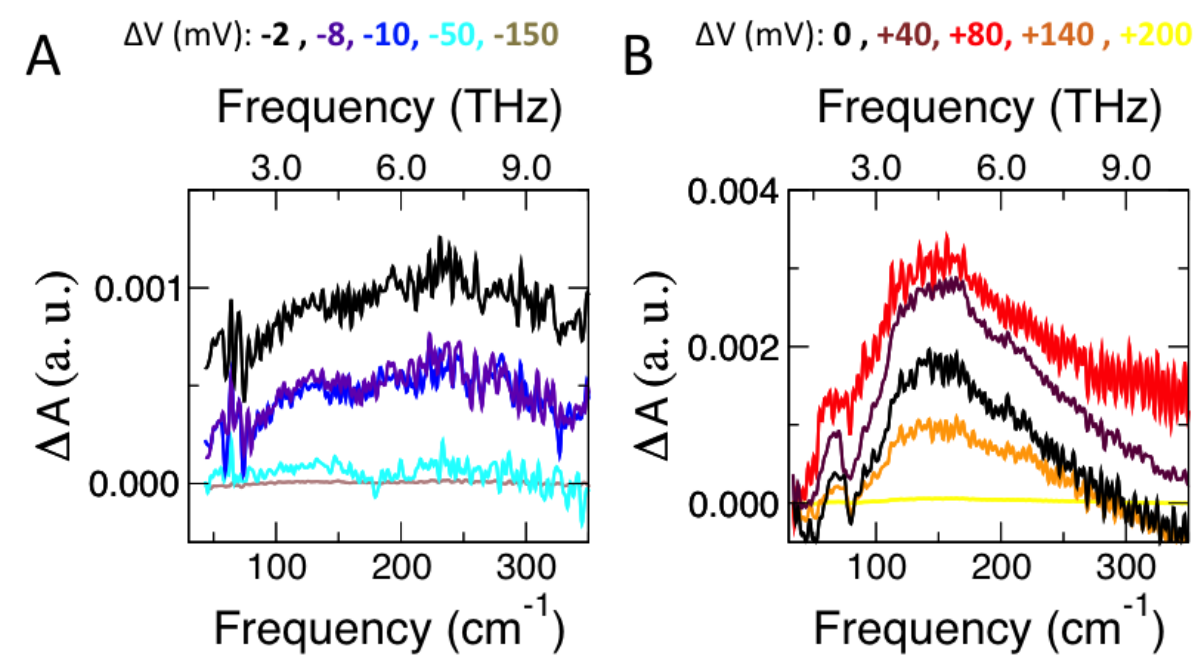

Figure 1: Absorption spectra as a function of the applied potential. A: spectra acquired during negative potential series for 5 selected voltages. Each spectrum is referenced to the spectrum acquired at $-150 \mathrm{mV}$. B: spectra acquired 
during positive potential series for 5 selected voltages. Each spectrum is referenced to the spectrum acquired at +200 $\mathrm{mV}$. The total set of spectra upon potential application is displayed in Fig. S7 and S9 of the SI.

In a nutshell, for both positive and negative potential series, a matrix $M$ is constructed, each row containing one of the measured difference spectra, and subsequently diagonalised in order to obtain an orthonormal basis set carrying the fundamental effect of the applied potential on the spectral features. In practice, each spectrum is rewritten as a linear combination of potential-independent partial spectral components (Principal Components, PCs) weighted by the potential-dependent scores (eigenvalues). While a standard fitting procedure requires assumptions on the number of the bands in a given spectral region, their position and shape, the advantage of PCA is that it does not depend on any a priori knowledge. However, the extracted spectral components still need to be associated to meaningful physical observables. This is done in Fig. 2, by comparing the spectral components obtained from the PCA with theoretically reconstructed spectral components. The scores obtained from the PCA are reported in Figure S1 in the SI. The theoretical spectra are obtained from classical molecular-dynamics (MD) simulations of positively and negatively charged Au-electrolyte interfaces (17 simulations with surface charge, $\sigma$, values from $-300 \mathrm{mC} / \mathrm{m}^{2}$ to $+300 \mathrm{mC} / \mathrm{m}^{2}$, see Methods Section for details).

As shown in Fig.2-A, a single spectral component is sufficient to describe the spectral changes with negative potentials, while two independent components are found for positive potentials (panels B and C). The residuals, obtained by subtracting the sum of the corresponding spectral components from the total spectrum (in Fig 1) at each potential (A, for negative potentials and $\mathrm{B}+\mathrm{C}$, for positive potentials), demonstrate that the low number of independent components is indeed sufficient to reproduce the full spectrum. 

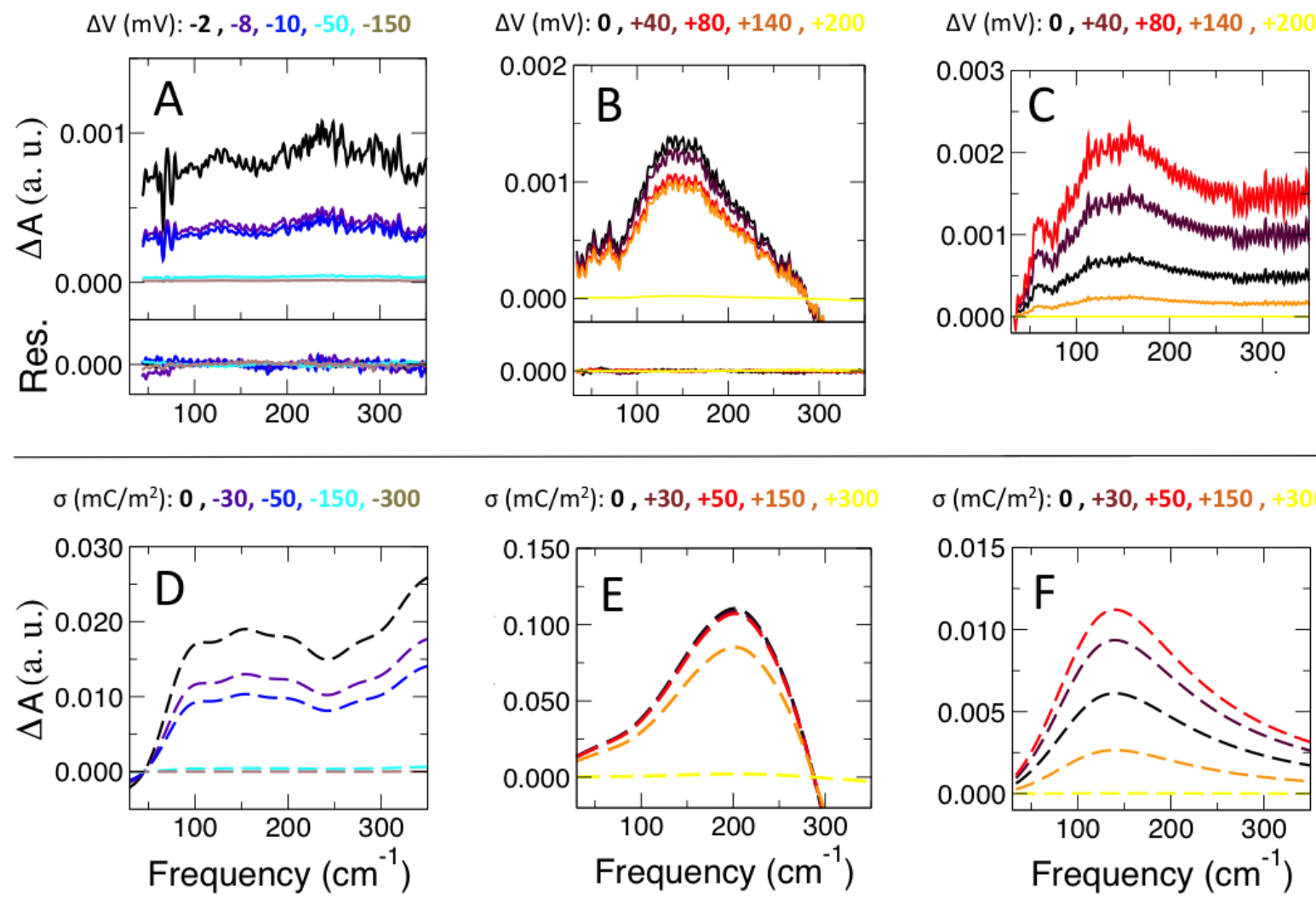

Figure 2: Experimental and theoretical partial spectral components as a function of negative and positive applied potential and surface charge. Top: (A) Partial spectra, attributed to $\mathrm{Na}^{+}$hydration, obtained multiplying the first principal component by the respective scores as a function of negative applied potential. Bottom: residuals derived from the subtraction of the partial spectra from the total spectra in Fig. 1-A. (B) Partial spectra, attributed to $\mathrm{Cl}^{-}$ hydration, obtained multiplying the second principal component by the respective scores as a function of positive applied potential. Bottom panel: residuals. (C) Partial spectra, attributed to the interfacial water network, obtained multiplying the first principal component by the respective scores as a function of positive applied potential. Bottom: (D) Simulated spectrum of hydrated $\mathrm{Na}^{+}$, from Ref. (39) multiplied by the variation in the number of hydration water around $\mathrm{Na}^{+}$cations as a function of the negative surface charge in the MD simulations. (E) simulated spectrum of hydrated $\mathrm{Cl}^{-}$ion, from ref (39) multiplied by the variation in the number of hydration water around $\mathrm{Cl}^{-}$as a function of the positive surface charge in the MD simulations. (F) Hydrogen bond (HB) stretch mode of water molecules hydrating a hydrophobic (alcohol) surfaces, as in (43), multiplied by the number of hydrogen bond of the 2D-HB network as a function of the positive surface charge in the MD simulations. 
In the following, we will analyze the results of the PCA by comparison with simulated spectral features. For each of the three PCs discussed above, we assign a calculated isolated spectral component and scale it by a surface charge dependent factor that is derived from either changes in the solvation environment of the ions or changes in the hydrogen bond network. This allows us to map the spectral changes to the atomistic processes at the EDL under applied bias. The spectral component for negative potentials in Fig.2-A is compared and assigned to the theoretical THz spectrum of solvated $\mathrm{Na}^{+}$as a function of the Au surface charge $(\sigma)$ in panel D. As detailed in the methods section, the spectra in panel D are obtained by multiplying the theoretical spectrum of solvated $\mathrm{Na}^{+}(39)$ by a weighting factor, chosen to be the variation of the number of hydration water molecules with $\sigma$ in the first solvation shell of $\mathrm{Na}^{+}$in the MD simulations. The calculated spectrum of solvated $\mathrm{Na}^{+}$is therefore associated with the theoretical ( $\sigma$-independent) principal component, and the variation of the number of hydration water molecules with $\sigma$ around $\mathrm{Na}^{+}$is used as a proxy for the $\sigma$-dependent score. Both experimental and theoretical spectra show a similar trend with negative applied potential/surface charge. Therefore, since the intensity in the theoretical spectra depends on the number of $\mathrm{Na}^{+}$hydration water molecules, an intensity increase/decrease in the experiments correlates directly with an increase/decrease in the number of hydration water around $\mathrm{Na}^{+}$cations at the interface.

We can hence assign the sharp intensity decrease detected in both the experimental and the theoretical spectra (panel A and D) to the (partial) depletion of the hydration layer of $\mathrm{Na}^{+}$at the interface, happening already at the smallest applied potentials/surface charges. When comparing the experimental spectral component in Fig. 2-A with the theoretical counterpart in Fig. 2-D we conclude that the $\Delta \mathrm{A}(\mathrm{v}, \mathrm{V})$ maximum at $\sim 250 \mathrm{~cm}^{-1}$ in Fig. 2-A does not arise from the hydration shell of $\mathrm{Na}^{+}$. It therefore has to be ascribed to a second contribution hidden in the experimental 
spectral component. This can occur when two (or more) spectral components share the same trend with the potential (i.e. same scores) and the PCA is not able to disentangle them. As discussed in more detail in the SI and since any ion contribution has been ruled out, this feature might be associated with the interfacial water network. Here, adlayer disordering at negative potentials (13) can induce a blue shift of the librational band (S3 in SI). In the methods section, this hypothesis is corroborated by the theoretical results showing that the $\mathrm{Na}^{+}$hydration and the interfacial water network disordering have a similar dependence on the negative charging of the Au surface (Fig. 5 $\mathrm{A} / \mathrm{C})$.

Intriguingly, a very different behaviour upon increase of the surface charge is observed for positive potentials. The experimental spectral component in Fig.2-B is assigned to hydrated $\mathrm{Cl}^{-}$ after comparison with the theoretical spectra in panel E. Analogously to the cationic case for negative bis potentials, the spectra in panel $\mathrm{E}$ are obtained by multiplying the theoretical spectrum of solvated $\mathrm{Cl}^{-}(39)$ by the variation of the number of hydration water molecules with $\sigma$ in the first solvation shell of $\mathrm{Cl}^{-}$in the $\mathrm{MD}$ simulations. Therefore, any change in intensity for this component can be associated to a change in the number of hydration waters solvating the anions at the interface. The spectral intensity is virtually constant in a large window of bias potentials/surface charges, implying that the $\mathrm{Cl}^{-}$coordination shell is almost unaffected, while the stripping off of the anions occurs only at the most positive values, when the intensity starts to decrease in both experimental and simulated spectra. In the experiments (panel B), the onset of such decrease is at $\Delta \mathrm{V}>140 \mathrm{mV}$.

Concomitantly, a second component is observed for positive potentials (panel C in Fig. 2), and assigned to the water network at the interface (see also Figure S5 in the SI for comparison with the $\mathrm{THz}$ absorption spectrum of bulk water). Strikingly, the center frequency of the 
underlying peak $\left(147 \pm 5 \mathrm{~cm}^{-1}\right.$, Figure S6 of the SI) resembles the hydration water band observed experimentally and theoretically in the hydration shell around hydrophobic alcohol chains (43). This band arises from a wrapped hydrogen-bond (HB) interfacial-water network, (44) reminiscent of the planar 2-dimensional-HB-network formed at the hydrophobic air-water interface (45) and at other planar interfaces such as graphene-water (65), where interfacial water HBs preferentially orient parallel to the surface. A similar in-plane interfacial water orientation has been reported for Au-water interfaces $(21,33)$. Importantly, this band is systematically red-shifted with respect to the $196 \mathrm{~cm}^{-1}$ band observed for bulk water $(33,41,47)$. We base this assignment on the comparison with the spectra in panel $\mathrm{F}$, which show the experimental spectrum of the HB stretching mode of water molecules hydrating a hydrophobic surface (41), weighted by the variation of the number of hydrogen bonds within the 2-dimension HB network as a function of $\sigma$.

The initial increase of the relative partial amplitudes for $\Delta \mathrm{V} \leq 80 \mathrm{mV}$ in Figure 2-C is therefore attributed to a strengthening of the interfacial water network, i.e. to an increase in the number of HBs formed parallel to the Au surface in the interfacial layer. By contrast, the decrease for higher $\Delta \mathrm{V}$ values reflects a partial breaking of the interfacial water structure. A comparison of the trends in panel B and C reveals that, at positive potentials, the spectral signature of the stripping off of the $\mathrm{Cl}^{-}$hydration shell starts to be detected (in panel B) only at the potential values when the interfacial water network is partially broken $(\mathrm{C})$.

Merging all the experimental results, we can infer that, for low positive potential values, the anions keep their full hydration layer and the interfacial HB-network is strengthened, while cations directly lose part of their hydration shell already at the lowest negative potential in our experimental window. This contrasts with what is described in a number of electrochemical models, in which $\mathrm{Na}^{+}$is expected to be more anchored to its hydration waters due to its higher 
charge density compared to $\mathrm{Cl}^{-}$(48). At high positive/negative potentials, the classical electrochemical picture is restored, and the hydration shells of both $\mathrm{Cl}^{-}$and $\mathrm{Na}^{+}$are depleted.

In order to confirm and rationalize the asymmetric stripping off of the $\mathrm{Cl}^{-}$and $\mathrm{Na}^{+}$hydration shells at the molecular level, we use a combination of classical MD simulations and ab initio calculations that naturally incorporate the PZC as their internal reference. While the (static) ab-initio calculations allow us to properly account for polarization and charge-transfer effects as a function of the applied voltage, the classical MD simulations allow us to simulate the low ionic concentrations used in the experiments and to perform a systematic investigation of the effects of surface charging, which are currently unfeasible by ab initio MD simulations with explicit solvent and applied bias. Therefore, these calculations allow us to investigate whether the asymmetric behaviour observed experimentally still holds true when either the applied bias (ab initio) or the surface charge (classical MD) is varied symmetrically around the Au PZC.

While the comparison with experimental results has been discussed earlier in the text, Figure 3 displays the resulting molecular picture of the fundamental microscopic electrochemical processes derived from the classical MD simulations. We report the average ion distribution as a function of the vertical distance from the Au surface for all investigated positive (left) and negative (right) $\sigma$ values. Both ion profiles show two peaks centered around 3 and $5 \AA$, corresponding to the inner and outer Helmholtz planes (IHP/OHP), respectively. The definition of the two planes is based on the distinct average coordination number of the ions. The density peak associated with the OHP identifies ions fully solvated within the water 2D-HB-Network located between 3 and 7 $\AA$ from the Au surface (see also sections S9-11 of SI), where they maintain their hydration layer intact, i.e. same as in the bulk. By contrast, both anions and cations in the IHP directly face the 
bare Au surface and lose on average one hydration water molecule. The change in the coordination of both ions with respect to the surface charge can hence be solely attributed to the way $\mathrm{Cl}^{-} / \mathrm{Na}^{+}$ populate the IHP. As shown in Figure 3, $\mathrm{Cl}^{-}$preferentially stays in the OHP for low and intermediate positive $\sigma$ and only migrates from the OHP to the IHP for the highest $\sigma>100 \mathrm{mC} / \mathrm{m}^{2}$, with a corresponding partial stripping off of its hydration shell. On the contrary, $\mathrm{Na}^{+}$ions immediately populate the IHP at the lowest $\sigma=-20 \mathrm{mC} / \mathrm{m}^{2}$ and start to occupy the OHP only when the IHP is saturated at around $\sigma=-70 \mathrm{mC} / \mathrm{m}^{2}$. When chloride ions are accumulated in the OHP, we observe an increase in the number of interfacial water molecules oriented with both $\mathrm{O}-\mathrm{H}$ groups parallel to the Au surface, leading to an increase in the number of parallel H-bonds forming the 2D-HB-network. This is shown in Figure 5-D (see also Figure S13 of the SI for water O-H groups orientation). This strengthening effect is lost once the anions are moved from the OHP to the IHP. Water molecules are then forced to align with respect to the high electrostatic field generated by the charged surface. Due to the reorientation, the number of in-plane H-Bonds formed between interfacial waters decreases, thereby weakening the 2D-HB-network. As long as the 2D-HBnetwork is still present at the interface $\left(\sigma \leq 100 \mathrm{mC} / \mathrm{m}^{2}\right)$, anion location in the OHP is therefore favoured by both ion-water and water-water interactions. On the contrary, the 2D-HB-network strengthening is not observed for negatively charged surfaces (Fig.5-C) for which interfacial water molecules orientation progressively changes from in-plane to pointing toward the surface. Thus, insertion of $\mathrm{Na}^{+}$into the IHP starts immediately upon application of a negative voltage as observed in the experiments and simulations. The reported changes in interfacial water structure are in good agreement with a recent combined experimental and ab-initio study (21), concluding that the topmost interfacial layer at the Au-water interface is mostly oriented parallel to the surface at $0 \mathrm{~V}$. When applying an increasingly negative potential, water was found to reorient first with one $\mathrm{OH}$, 
and finally with both $\mathrm{OH}$ pointing toward the surface (Figure S12). Such behaviour has been also described by MD simulations in Ref. (13), revealing that under a negative bias the re-orientation of water molecules with $\mathrm{H}$ atoms toward the gold surface disrupts the HB-network in the interfacial layer. Furthermore, our results on the in-plane orientation of water that persists longer under positive surface charging supports the idea of an asymmetric reorientation of water at positive/negative electrodes as described in Refs. $(13,49)$. The novel result provided here is that the building-up of an in-plane oriented interfacial layer exclusively at positively charged gold surfaces causes anions and cations to migrate differently when positive/negative potentials are applied.

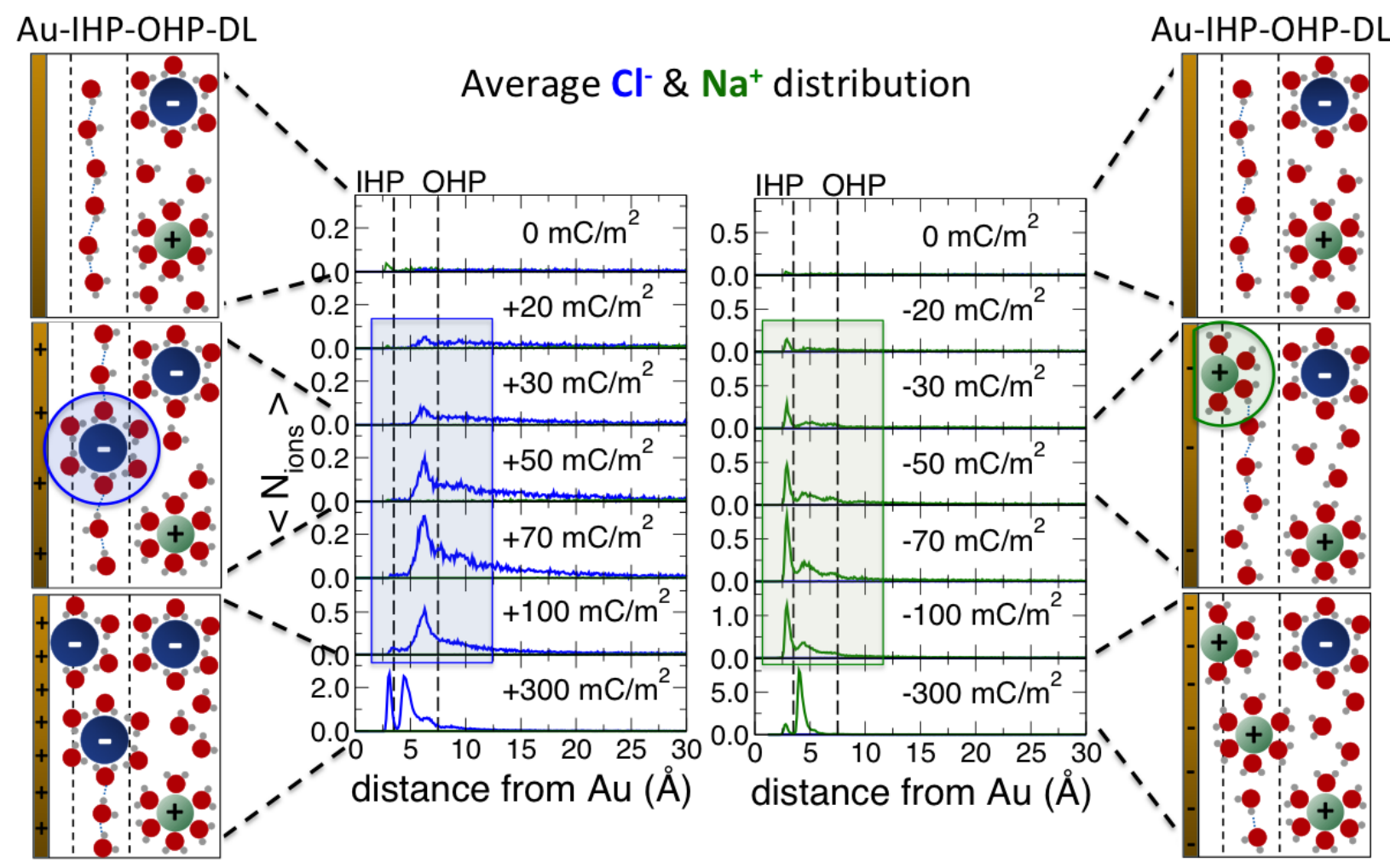

Figure 3: Microscopic view of the double layer formation at the Au electrode. Average number of ions $\left(\mathrm{Cl}^{-}\right.$in blue, $\mathrm{Na}^{+}$in green) as a function of the distance from a positively (left) and negatively (right) charged Au surface. Dashed lines mark the borders of the IHP and OHP. Left and right: six key scenarios for ion distributions at zero, low 
and high surface charge values (from top to bottom). The average number of ions in the IHP and OHP, as obtained from integration of the ions distributions in each of the two regions, can be found in Table S2 of the SI.

However, these calculations do not account for polarization and chemical changes induced by the ions and the applied bias. Energy decomposition analysis of charged gold cluster models (Figure 4-A and section S16) demonstrates that polarization plays a role but is independent of the charge. These calculations also reveal charge-transfer from the chloride ion to the gold cluster that is completely absent in the $\mathrm{Na}^{+}$-Au interaction. However, the fact that these effects are independent of cluster charge rules them out as the source of the different behavior of $\mathrm{Na}^{+}$and $\mathrm{Cl}^{-}$at the electrochemical double-layer. Grand-canonical (in electrons) periodic slab calculations on a $\mathrm{Au}(100)$ surface additionally confirm that the polarization of the electrode as measured by the change of number of electrons present in the metal slab is largely independent of the applied bias (Figure 4-B and section S17) in the experimentally accessed range. In addition, a microsolvation analysis (section S18) demonstrates that $\mathrm{Cl}^{-}$prefers a surface-like asymmetric microsolvation pattern as is present in the hydrophobic OHP ( $c f r$ ref. 33), whereas $\mathrm{Na}^{+}$prefers to be symmetrically solvated which can be achieved at the IHP by replacing one or two water molecules with negatively charged Au atoms. All in all, the quantum-chemical calculations support the results obtained from experiment and the classical MD simulations and hint that the deficiencies of a classical fixed charge model do not lead to biased conclusions in the current study. 

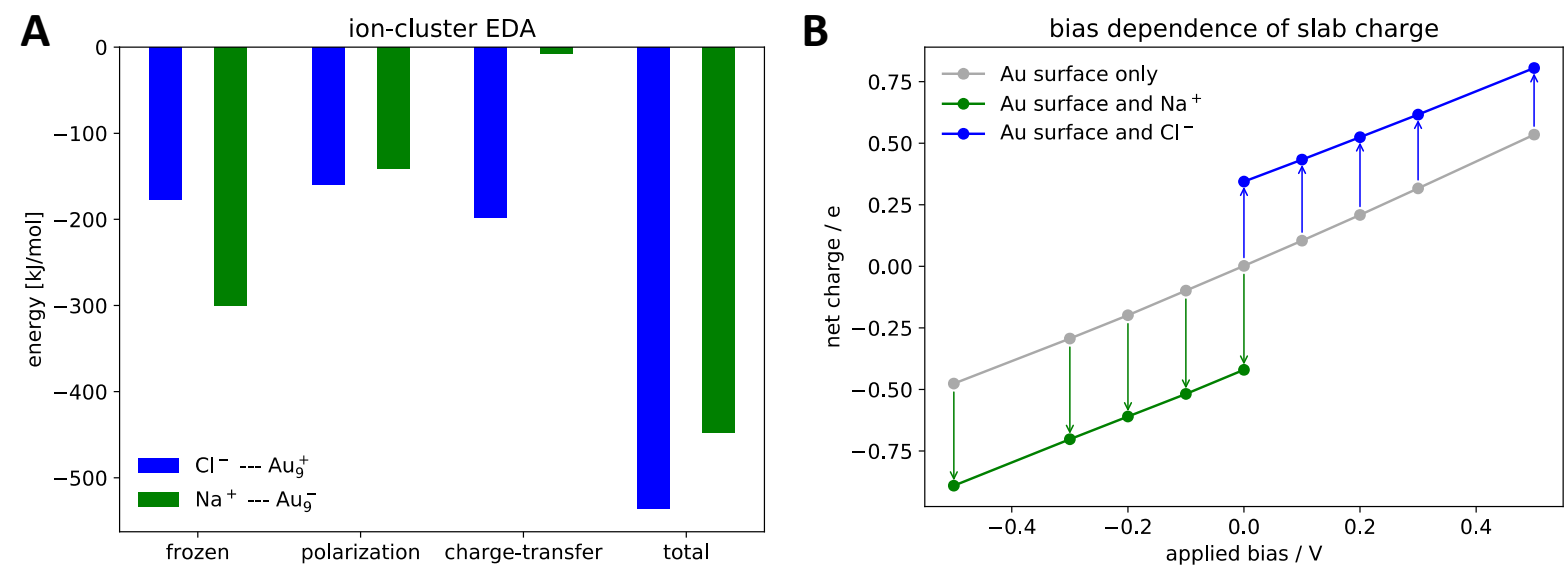

Figure 4: Results of electronic structure calculations. Panel A compares the different contributions to the interaction energy of the $\mathrm{Na}^{+}$(green) and $\mathrm{Cl}^{-}$(blue) ion with a negatively and positively charged Aug cluster model, respectively, as calculated with $\omega \mathrm{B} 97 \mathrm{X}-\mathrm{V} / \mathrm{def} 2-\mathrm{TZVPD}$. While the polarization term is of equal magnitude, charge-transfer is completely absent in case of $\mathrm{Na}^{+}$, whereas it contributes to the interaction energy for the $\mathrm{Cl}^{-}$case. Panel $\mathrm{B}$ shows the net charge of a grand-canonical periodic slab model with varying bias potential referenced against the computed potential of zero charge. The green (blue) data points show the corresponding decrease (increase) of the net charge upon $\mathrm{Na}^{+}\left(\mathrm{Cl}^{-}\right)$binding. The change of this polarization effect is negligible in the experimental bias range $(-0.15-$ $0.2 \mathrm{~V}$ ) as indicated by the constant length of the colored arrows.

In conclusion, by a novel $\mathrm{THz}$ spectro-electrochemical approach combined with molecular dynamics simulations, we have directly probed the solvation/desolvation processes at the $\mathrm{Au}$ surface under in-operando electrochemical conditions. Our results have dissected at the microscopic level the stripping off of the hydration shells of the electrolyte ions as well as the associated changes in the interfacial water HB-network. Our approach can now be used to investigate the crucial role of water in mediating other interfacial processes at metal as well as semiconductor/electrolyte interfaces. This new view will impact the understanding and optimization of electrochemical processes for technological applications. 


\section{Methods}

\section{THz spectro-electrochemical measurement}

THz-Far infrared (THz/FIR) absorption spectra of $10 \mathrm{mM} \mathrm{NaCl}$ (Sigma-Aldrich, purity $\geq 98 \%$ ) were recorded in the frequency range $50-350 \mathrm{~cm}^{-1}$ with $2 \mathrm{~cm}^{-1}$ resolution. We employed a vacuumevacuated $\left(10^{-5}\right.$ mbar) Fourier transform infrared spectrometer (FTIR, IFS 125, Bruker) with an external 4.2 K He-cooled bolometer (Infrared Laboratories, Inc., Tucson, USA) for the detection

of the synchrotron light (at the beamline AILES, Soleil). The source has a flux of $5 \cdot 10^{13}$ photons $/ \mathrm{s} / 0.1 \%$ bandwidth at $100 \mathrm{~cm}^{-1}$. Each single spectrum is the average of 128 scans recorded at $40 \mathrm{kHz}$ at $25^{\circ} \mathrm{C}$. To apply the constant potential, we did use a potentiostat (PalmSens 4, PalmSens BV), shortcutting the exit of the RE on the CE.

From each spectrum at the potential $\mathrm{V}_{\mathrm{i}}$, the previously recorded spectrum at the potential $\mathrm{V}_{\text {ref }}$ is subtracted. The difference spectra are then decomposed by Principal Component Analysis (PCA) into independent spectral components (PCs), based on their distinct response to the potential increase/decrease. The changes in the total spectrum are then attributed to voltage dependent changes of the respective partial spectra. One spectral component for the negative potential series and two spectral components for the positive one are sufficient to describe the voltage dependent changes in the spectral dataset. Thus, the acquired data sets are written as $\Delta \mathrm{A}_{+}(v, \mathrm{~V})=$ scores $_{1, \text { neg }}(\mathrm{V}) \cdot \mathrm{PC}_{1, \mathrm{neg}}(v)$ and $\Delta \mathrm{A}_{\text {pos }}(v, \mathrm{~V})=\operatorname{scores}_{1, \operatorname{pos}}(\mathrm{V}) \cdot \mathrm{PC}_{1, \operatorname{pos}}(v)+\operatorname{scores}_{2, \operatorname{pos}}(\mathrm{V}) \cdot \mathrm{PC}_{2, \operatorname{pos}}(v)$ for negative and positive potentials, respectively.

\section{Molecular Dynamics simulations}

Classical simulations were performed using the LAMMPS (50) code to simulate 17 aqueous solutions (17486 water molecules) of $20 \mathrm{mM} \mathrm{NaCl}$ confined between parallel charged gold $\mathrm{Au}$ (100) walls with imposed $298 \mathrm{~K}$ constant temperature to water and ions all along the simulation. 
The simulated surface charge values are: $0, \pm 20, \pm 30, \pm 50, \pm 70, \pm 100, \pm 170, \pm 240, \pm 300 \mathrm{mC} / \mathrm{m}^{2}$. Since no ion-pairing interactions are detected in the MD simulations with $20 \mathrm{mM} \mathrm{NaCl}(<0.01 \%$ probability to have an ion pair), no differences are reasonably expected between $10 \mathrm{mM}$ (experimental concentration) and $20 \mathrm{mM}$ concentrations, which are relevant to the conclusions of this work. The constant charge is imposed on the topmost atomic layer. The water $+\mathrm{NaCl}$ system was described using the force field developed by Kann and Skinner (51), based on the TIP4P/2005 model of water and employing rescaled (by 0.85) charges for ions. Charge rescaling compensates for the underestimated permittivity of TIP4P/2005 water, and effectively describes ion polarizability and charge delocalisation of solvated ions (51). We systematically rescaled the surface charge with the same ratio as the one applied to ions. The interactions between $\mathrm{Au}$ atoms and liquid atoms were treated using the Lennard-Jones parameters introduced by Heinz et al. (52) and Lorentz-Berthelot mixing rules. 3-Dimensional periodic boundary conditions were applied with lateral ab-dimensions of $56.213 \AA$ and counterions were added to the system to ensure electroneutrality. To impose a pressure of $1 \mathrm{~atm}$, we used the top wall as a piston until an equilibrium height was reached, and we fixed the top wall height at its equilibrium position for the rest of the equilibration and for the production runs. The same simulation protocol with an equilibration run of $36 \mathrm{~ns}$ (with a timestep of $1 \mathrm{fs}$ ) followed by a production run of $36 \mathrm{~ns}$ (with a timestep of $2 \mathrm{fs}$ ) has been systematically followed. The total simulation time, including the equilibration period where the upper solid surface is used as a piston, is about $90-100 \mathrm{~ns}$ for each simulation. We carefully checked that at the end of the equilibration time ions (and water) were correctly distributed in the simulation box with correct density profiles.

The differences in the total $\mathrm{Cl}-\mathrm{O}$ and $\mathrm{Na}-\mathrm{O}$ coordination numbers $\left(\Delta \mathrm{N}_{\mathrm{Cl}-\mathrm{O}}(\sigma)\right.$ and $\Delta \mathrm{N}_{\mathrm{Na}-\mathrm{O}}(\sigma)$, respectively) with respect to the positive and negative surface charging, used as "theoretical 
scores" to calculate the spectra in Figure 2-D/E are derived as follows:

$$
\Delta \mathrm{N}_{\text {ion }}(\sigma)=\mathrm{N}_{\text {ion }}{ }^{\text {int }}(\sigma)\left(\operatorname{coord}_{\text {ion-o }}{ }^{\text {int }}(\sigma)-\operatorname{coord}_{\text {ion-O }} \text { bulk }^{\text {but }}\right)
$$

where $\mathrm{N}_{\text {ion }}{ }^{\text {int }}(\sigma)$ is the number of $\mathrm{Cl} / \mathrm{Na}$ in the interfacial layer (i.e. in the IHP+OHP), coordion$\mathrm{O}^{\mathrm{int}}(\sigma)$ is the average $\mathrm{Cl} / \mathrm{Na}$ coordination number in the interfacial layer at a given $\sigma$ value, and coord $_{\text {ion- }} \mathrm{O}^{\text {bulk }}$ is the average $\mathrm{Cl} / \mathrm{Na}$ coordination in bulk. The differences in the total $\mathrm{Cl}-\mathrm{O}$ and NaO coordination numbers (shown in Figure 5-A/B) represent the variation in the coordination of all ions at the interface with respect to their coordination in the bulk. In order to obtain the theoretical spectra in Figure 2-D/E, the theoretical spectra of solvated $\mathrm{Na}^{+}$and $\mathrm{Cl}^{-}$from Ref. (39) are scaled by these scores. The solvated $\mathrm{Na}^{+}$spectrum is the sum of the cation autocorrelation and its crosscorrelations with water molecules in the first and second solvation shell $\left(\Delta \mathrm{C}_{\text {ion }}\right)$ and the autocorrelation of the first-shell water molecules $\left(\Delta \mathrm{C}^{1}\right)$. The solvated $\mathrm{Cl}^{-}$spectrum arises from the H-bonds between the first and the second shell $\left(\mathrm{C}_{12}{ }^{\mathrm{HB}}\right)$. The theoretical scores used to calculate the spectra in Figure 2-F are related to the strength of the 2D-HB-Network, measured as the average number of HBs formed in between water molecules in the topmost interfacial layer and oriented parallelly to the surface $\left(\mathrm{HBs}_{2 \mathrm{DN}} /\right.$ molecule). The results obtained for both positive and negative surface charge values are plotted in Figure 5-C/D. Water-water H-Bonds are defined using a mixed distance-angle criterion, with the H-Bond O-O distance cut-off of $3.2 \AA$ and the H-Bond $\mathrm{O}-\mathrm{H}^{\cdots} \mathrm{O}$ angle in the range $[140-220]^{\circ}$. Different criteria (e.g. H-Bond O-O distance cut-off of $3.5 \AA$ and $\mathrm{H}-\mathrm{Bond} \mathrm{O}-\mathrm{H} \cdots \mathrm{O}$ angle in the range $\left.[150-210]^{\circ}\right)$ lead to the same trends as reported in Figure 5. Moreover, the changes in the water network upon negative surface charging (panel C) show a similar trend as $\Delta \mathrm{N}_{\mathrm{Na}-\mathrm{o}}$. Therefore, PCA is not able to separate the possible spectral contribution of the change in the interfacial water network from the $\mathrm{Na}^{+}$hydration. This observation corroborates the hypothesis that the feature at around $250 \mathrm{~cm}^{-1}$ in the spectra of Figure 
2-A arises from a water-related contribution with the same potential dependence as the $\mathrm{Na}^{+}$ hydration fingerprint.
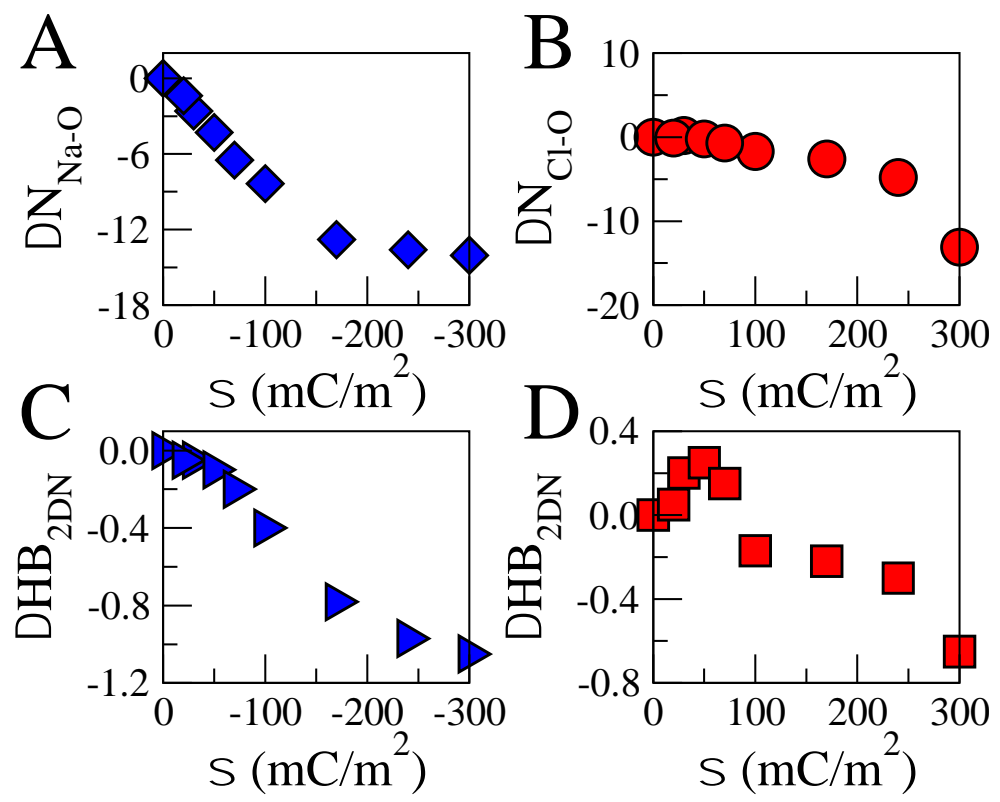

Figure 5: Theoretical scores used to calculate the spectra shown in Figure 2 (lower panels) (A) Differences in the total $\mathrm{Na}-\mathrm{O}$ coordination numbers with respect to the positive surface charging. (B) Differences in the total $\mathrm{Cl}-\mathrm{O}$ coordination numbers with respect to the negative surface charging. (C) Average number of HBs per molecule formed in between water molecules in the topmost interfacial layer and oriented parallelly to the surface for positive surface charging. (D) Average number of HBs per molecule formed in between water molecules in the topmost interfacial layer and oriented parallelly to the surface for negative surface charging.

\section{Electronic Structure Calculations}

All density functional calculations for the cluster models were carried out with the Q-Chem electronic structure package (53). We applied the RPBE functional (54) for consistency with the periodic slab calculations and the $\omega \mathrm{B} 97 \mathrm{X}-\mathrm{V}$ functional (55) for comparison. The density functional theory calculations for the periodic slab models were carried out on the $\mathrm{Au}$ (100) surface using the Vienna Ab Initio Simulation Package (VASP) (56,57). The surface unit cell (periodically extending 
in the $x$-and $y$-directions) was designed as four layers of eight Au atoms, with a lattice constant of $4.0782 \AA$ and $20 \AA$ spacing (in the $z$-direction) between two images. A $k$-point sampling of the Brillouin zone was achieved using the $4 \times 4 \times 1$ Monkhorst-Pack mesh. The implicit aqueous solvent and the electrolyte were implemented with a dielectric constant $\varepsilon_{r}=78.4$ and a DebyeHückel length $\lambda_{b}=9.61 \AA$.

More details are found in the Supporting Information.

\section{References}

[1] G. Glenk, S. Reichelstein, Economics of converting renewable power to hydrogen. Nature Energy, 4, 216-222 (2019).

[2] N. S. Lewis, Toward Cost-Effective Solar Energy Use. Science 315, 798-801 (2007).

[3] K. Maeda, K. Teramura, D. Lu, T. Takata, N. Saito, Y. Inoue, K. Domen, Photocatalyst releasing hydrogen from water. Nature 440, 295 (2006).

[4] F. Studt, M. Behrens, E. L. Kunkes, N. Thomas, S. Zander, A. Tarasov, J. Schumann, E. Frei, J. B.Varley, F. Abild-Pedersen, J. K. Nørskov, R. Schlögl, The Mechanism of $\mathrm{CO}$ and $\mathrm{CO}_{2}$ Hydrogenation to Methanol over Cu-Based Catalysts. ChemCatChem, 7, 1105-11 (2015).

[5] W. J. Albery, G. J. O'Shea, A. L. Smith, Interpretation and use of Mott-Schottky plots at the semiconductor/electrolyte interface, J. Chem. Soc., Faraday Trans., 92, $4083-85$ (1996).

[6] K. B. Oldham, A Gouy-Chapman-Stern model of the double layer at a (metal)/(ionic liquid) interface. J. Electroanal. Chem. 613, 131-138 (2008).

[7] R. Grassi, A. Daghetti, S. Trasatti, Application of the Gouy-Chapman-Stern-Grahame model of the electrical double layer to the determination of single ion activities of KF aqueous solutions. 
J. Electroanal. Chem. 226, 341-349 (1987).

[8] T. B. Kinraide, Use of a Gouy-Chapman-Stern model for membrane-surface electrical potential to interpret some features of mineral rhizotoxicity, Plant Physiol. 106, 1583-92 (1994).

[9] Stern, O. The theory of the electrolytic double shift. Z. Eleckrochem. Angew. Phys. Chem. 30, 508-516 (1924).

[10] R.D. Armstrong, B.R. Horrocks, The double layer structure at the metal-solid electrolyte interface, Solid State Ionics, 94, 181-187 (1997).

[11] P. E. Ohno, H. Chang, A. P. Spencer, Y. Liu, M. D. Boamah, H. Wang, F. M. Geiger, Beyond the Gouy-Chapman Model with Heterodyne-Detected Second Harmonic Generation. J. Phys. Chem. Lett. 10, 2328-34 (2019).

[12] C. Merlet, D. T. Limmer, M. Salanne, R. Van Roij, P. A. Madden, D. Chandler, B. Rotenberg. The electric double layer has a life of its own. J. Phys. Chem. C 118, 18291-98 (2014).

[13] A. Willard, S. K. Reed, P. A. Madden, D. Chandler, Water at an electrochemical interface - a simulation study. Faraday Discuss. 141, 423-441 (2009).

[14] M. Mucha, T. Frigato, L. M. Levering, H. C. Allen, D. J. Tobias, L. X. Dang, P. Jungwirth, Unified molecular picture of the surfaces of aqueous acid, base, and salt solutions. J. Phys. Chem. $B$ 109, 7617-23 (2005).

[15] A. S. Thomas, A. H. Elcock, Molecular Dynamics Simulations of Hydrophobic Associations in Aqueous Salt Solutions Indicate a Connection between Water Hydrogen Bonding and the Hofmeister Effect. J. Am. Chem. Soc. 129, 14887-98 (2007).

[16] J. Rossmeisl, E. Skúlason, M. E. Björketun, V. Tripkovic, J. K.Nørskov, Modeling the electrified solid-liquid interface. Chem. Phys. Lett. 466, 68-71 (2008).

[17] L. Joly, C. Ybert, E. Trizac, L. Bocquet, Hydrodynamics within the electric double layer on 
slipping surfaces. Phys. Rev. Lett. 93, 257805 (2004).

[18] J.-J. Velasco-Velez, C. H. Wu, T. A. Pascal, L. F. Wan, J. Guo, D. Prendergast, M. Salmeron, The structure of interfacial water on gold electrodes studied by x-ray absorption spectroscopy. Science 346, 831-834 (2014).

[19] M. Favaro, B. Jeong, P. N. Ross, J. Yano, Z. Hussain, Z. Liu, E.J. Crumlin, Unravelling the electrochemical double layer by direct probing of the solid/liquid interface. Nat. Comm. 7, 12695 (2016).

[20] W.-T. Liu, Y.R. Shen, In situ sum-frequency vibrational spectroscopy of electrochemical interfaces with surface plasmon resonance. Proc. Nat. Am. Soc. 111, 1293-97 (2014).

[21] C.Y. Li, J. B. Le, Y.H. Wang, S. Chen, Z. L. Yang, J.F. Li, J. Cheng, Z.Q. Tian, In situ probing electrified interfacial water structures at atomically flat surfaces. Nature Materials 18, 697-701 (2019).

[22] Y. Shingaya, K.Hirota, H. Ogasawara, M. Ito, Infrared spectroscopic study of electric double layers on $\operatorname{Pt}(111)$ under electrode reactions in a sulfuric acid solution. J. Electrochem. Chem. 409, 103-108 (1996).

[23] Z. D. Schultz, S. K. Shaw, A. A. Gewirth, Potential dependent organization of water at the electrified metal-liquid interface. J. Am. Chem. Soc. 127, 15916-22 (2005).

[24] A. Yamakata, E. Soeta, T. Ishiyama, M. Osawa, and A. Morita, Real-time observation of the destruction of hydration shells under electrochemical force. J. Am. Chem. Soc. 135, 15033-39 (2013).

[25] M. Brown, Z. Abbas, A. Kleibert, R. G. Green, A. Goel, S. May, and T. M. Squires, Determination of Surface Potential and Electrical Double-Layer Structure at the Aqueous Electrolyte-Nanoparticle Interface. Phys. Rev. X 6, 011007 (2016). 
[26] J. Chmiola, C. Largeot, P.-L. Taberna, P. Simon, Y. Gogotsi, Desolvation of Ions in Subnanometer Pores and Its Effect on Capacitance and Double-Layer Theory. Angew. Chem. Int. Ed. 120, 3440-43 (2008).

[27] M. V. Fedorov, A. A. Kornyshev, Towards understanding the structure and capacitance of electrical double layer in ionic liquids. Electrochemical Acta, 53, 6835-6840 (2008).

[28] D. J. Tobias, J.C. Hemminger, Chemistry. Getting specific about specific ion effects. Science 319, 1197-8 (2008).

[29] G. V. Franks, Zeta potential and yield stress of silica suspensions. J. Coll. Int. Sci. 249, 44-51 (2002).

[30] K. Tschulik, W. Cheng, C. Batchelor-McAuley, S. Murphy, D. Omanović, R. G. Compton, Non-Invasive Probing of Nanoparticle Electrostatics. ChemElectroChem 2, 112-118 (2015).

[31] A.M. Gardner, K.H. Saeed, A.J. Cowan, Vibrational sum-frequency generation spectroscopy of electrode surfaces: studying the mechanisms of sustainable fuel generation and utilisation. Phys.Chem.Chem.Phys. 21, 12067-12086 (2019).

[32] H. Shi, Z. Cai, J. Patrow, B. Zhao, Y. Wang, Y. Wang, A. Benderskii, J. Dawlaty, S.B. Cronin, Monitoring Local Electric Fields at Electrode Surfaces Using Surface Enhanced Raman Scattering-Based Stark-Shift Spectroscopy during Hydrogen Evolution Reactions. ACS Appl. Mater. Interfaces, 10, 33678-33683 (2018).

[33] D. T. Limmer, A. P. Willard, P. Madden, D. Chandler, Hydration of metal surfaces can be dynamically heterogeneous and hydrophobic. Proc. Nat. Am. Soc. 110, 4200-4205 (2013).

[34] M. Heyden, J. Sun, S. Funkner, G. Mathias, H. Forbert, M. Havenith, D. Marx, Dissecting the THz spectrum of liquid water from first principles via correlations in time and space. Proc. Nat. Am. Soc. 107, 12068-73 (2010). 
[35] H.R. Zelsmann, Temperature dependence of the optical constants for liquid $\mathrm{H}_{2} \mathrm{O}$ and $\mathrm{D}_{2} \mathrm{O}$ in the far IR region, J. Mol. Struct., 350, 95-114 (1995).

[36] G. E. Walrafen, Raman Spectral Studies of the Effects of Temperature on Water and Electrolyte Solutions, J. Chem. Phys. 44, 1546 (1966).

[37] J. B. Hasted, S. K. Husain, F. A. M. Frescura, J. R. Birch, Far-infrared absorption in liquid water, Chem. Phys. Lett., 118, 622-25 (1985).

[38] G. Schwaab, F. Sebastiani, and M. Havenith, Ion Hydration and Ion Pairing as Probed by THz Spectroscopy, Angew. Chem .Int. Ed. 58, 3000-13 (2019).

[39] P. Schienbein, G. Schwaab, H. Forbert, M. Havenith, D. Marx, Correlations in the solutesolvent dynamics reach beyond the first hydration shell of ions. J. Phys. Chem. Lett. 8, 2373-80 (2017).

[40] C. T. Nemes, J. R. Swierk, and C. A. Schmuttenmaer, A Terahertz-Transparent Electrochemical Cell for In Situ Terahertz Spectroelectrochemistry, Anal. Chem. 90, 7, 4389-96 (2018).

[41] N. Vita, J. B. Brubach, R. Hienerwadel, N. Bremond, D. Berthomieu, P. Roy, C. Berthomieu, Electrochemically-induced far-Infrared difference spectroscopy on metalloproteins using advanced synchrotron technology. Anal. Chem. 85, 2891-98 (2013).

[42] Y. Wang, E. Gordon, H. Ren, Mapping the Potential of Zero Charge and Electrocatalytic Activity of Metal-Electrolyte Interface via a Grain-by-Grain Approach, Anal. Chem. 92, 2859$2865(2020)$.

[43] a) F. Böhm, G. Schwaab, M. Havenith, Mapping Hydration Water around Alcohol Chains by THz Calorimetry. Angew. Chem. Int. Ed. 56, 9981-85 (2017). b) V. Conti Nibali, S. Pezzotti, F. Sebastiani, D. Galimberti, G. Schwaab, M. Heyden, M.-P. Gaigeot, M. Havenith, J. Phys. Chem. 
Lett. 11, 4809-16 (2020).

[44] S. Funke, F. Sebastiani, G. Schwaab, M. Havenith, Spectroscopic Fingerprints in the low frequency spectrum of ice (Ih), clathrate hydrates, supercooled water and hydrophobic hydration reveal similarities in the hydrogen bond network motifs. J. Chem. Phys. 50, 224505 (2019).

[45] S. Pezzotti, D. R. Galimberti, M. P. Gaigeot, 2D-H-Bond-Network as the Topmost Skin to the Air-Water Interface. J. Phys. Chem. Lett. 8, 3133-41 (2017).

[46] B. Grosjean, M.-L. Bocquet, R. Vuilleumier, Versatile electrification of two-dimensional nanomaterials in water, Nat.Comm., 10, 1656 (2019).

[47] S. Pezzotti, A. Serva, M. P. Gaigeot, 2D-HB-Network at the air-water interface: a structural and dynamical characterization by means of ab-initio and classical molecular dynamics simulations. J. Chem. Phys. 148, 174701 (2018).

[48] F. Anson, Patterns of Ionic and Molecular Adsorption at Electrodes, Accounts of Chemical Research, 8, 400-407 (1975).

[49] Y. Zhang, H. B. de Aguiar, J. T. Hynes, D. Laage, Water Structure, Dynamics, and SumFrequency Generation Spectra at Electrified Graphene Interfaces. J. Chem. Phys. Lett. 11, 624-631 (2020).

[50] S. Plimpton, Fast Parallel Algorithms for Short Range Molecular Dynamics. J. Chem. Phys. $117,1-19(1995)$.

[51] Z. R. Kann, J. L. Skinner, A scaled-ionic-charge simulation model that reproduces enhanced and suppressed water diffusion in aqueous salt solutions. J. Chem. Phys. 141, 104507 (2014).

[52] H. Heinz, R. A. Vaia, B. L. Farmer, R. R. Naik, Accurate Simulation of Surfaces and Interfaces of Face-Centered Cubic Metals Using 12-6 and 9-6 Lennard-Jones Potentials. J. Phys. Chem. C 
$112,17281-90(2008)$.

[53] Y. Shao, Z. Gan. E. Epifanovsky, A. T. Gilbert, M. Wormit et al., Advances in molecular quantum chemistry contained in the Q-Chem 4 program package. Mol. Phys. 113, 184-215 (2015).

[54] B. Hammer, L. B. Hansen, J. K. Nørskov, Improved adsorption energetics within density functional theory using revised Perdew-Burke-Ernzerhof functionals. Phys. Rev. B 59, 7413-21 (1999).

[55] N. Mardirossian, M. Head-Gordon, $\omega B 97 X-V$ : A 10-parameter, range-separated hybrid, generalized gradient approximation density functional with nonlocal correlation, designed by a survival-of-the-fittest strategy, Phys. Chem. Chem. Phys. 16, 9904-24 (2014).

[56] G. Kresse, J. Furthmüller, Efficient iterative schemes for ab initio total-energy calculations using a plane-wave basis set, Phys. Rev. 54, 11169-86 (1996).

[57] G. Kresse, J. Furthmüller, Efficiency of ab-initio total energy calculations for metals and semiconductors using a plane-wave basis set, Comp. Mat. Sci., 6, 15-50 (1996).

\section{ACKNOWLEDGMENTS}

M. Havenith acknowledges financial support by the ERC Advanced Grant $695437 \mathrm{THz}-$ Calorimetry. This work is part of the Cluster of Excellence RESOLV (EXC 2033) funded by the 
Deutsche Forschungsgemeinschaft (DFG, German Research Foundation) under Germany's Excellence Strategy - EXC 2033 - 390677874 - RESOLV and the IMPRS-RECHARGE program. S.F., T.O., M.H., and K.T. acknowledge funding by the Research Training Group "Confinementcontrolled Chemistry"(GRK2376 / 331085229) by the Deutsche Forschungsgemeinsschaft (DFG). S.P. and M.P.G. acknowledge that this work was done under funding by ANR DYNAWIN Grant No. 14-CE35-0011-01. HPC resources from GENCI-France Grant No. 072484 (CINES/IDRIS/TGCC) are acknowledged. KT acknowledges funding by the Ministry of Innovation, Science and Research of North Rhine-Westphalia ("NRW Rückkehrerprogramm"). CJS and MHG acknowledge support from the U.S. Department of Energy, Office of Science, Office of Advanced Scientific Computing, and Office of Basic Energy Sciences, via the Scientific Discovery through Advanced Computing (SciDAC) program, with additional support from CALSOLV, and for CJS from an Early Postdoc.Mobility fellowship from the Swiss National Science Foundation. ZL was funded by the Joint Center for Artificial Photosynthesis, a DOE Energy Innovation Hub, supported through the Office of Science of the U.S. Department of Energy under Award Number DE-SC0004993. The authors thank Prof L. Joly from ILM-University of Lyon-France and Prof. J. M. Lisy from University of Illinois-US for discussions and advice. We further thank Louis Henri Michel Godeffroy for additional measurements regarding the $\mathrm{Ag} / \mathrm{AgCl}$ reference electrode. 